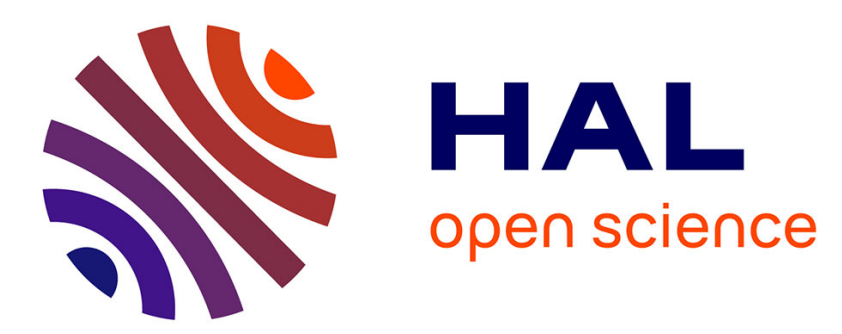

\title{
FPGA implementation of a low-cost method for tracking the resonance frequency and the quality factro of MEMS sensors
}

Farbod Ghassemi, Maira Possas, Gilles Amendola, Jérome Juillard

\section{- To cite this version:}

Farbod Ghassemi, Maira Possas, Gilles Amendola, Jérome Juillard. FPGA implementation of a lowcost method for tracking the resonance frequency and the quality factro of MEMS sensors. 2012 IEEE SENSORS, Oct 2012, Taipei, Taiwan. CD-ROM Proceedings (4 p.), 10.1109/ICSENS.2012.6411033 . hal-00778760

\section{HAL Id: hal-00778760 \\ https://hal-centralesupelec.archives-ouvertes.fr/hal-00778760}

Submitted on 21 Jan 2013

HAL is a multi-disciplinary open access archive for the deposit and dissemination of scientific research documents, whether they are published or not. The documents may come from teaching and research institutions in France or abroad, or from public or private research centers.
L'archive ouverte pluridisciplinaire HAL, est destinée au dépôt et à la diffusion de documents scientifiques de niveau recherche, publiés ou non, émanant des établissements d'enseignement et de recherche français ou étrangers, des laboratoires publics ou privés. 


\section{FPGA implementation of a low-cost method for tracking the resonance frequency and the quality factor of MEMS sensors}

\author{
Farbod Ghassemi, Maira Possas and Gilles Amendola \\ SYLE Department \\ ESIEE Paris \\ Noisy-Le-Grand, France
}

\author{
Jérôme Juillard \\ SSE \\ SUPELEC \\ Gif-sur-Yvette, France
}

\begin{abstract}
This paper reports the hardware implementation of a low-cost parameter estimation method (LIMBO) and describes its use for tracking the parameters of a resonant MEMS sensor. We show how this method can be used to identify the impulse response (IR) of a sensor with minimal A/D-D/A requirements. Based on this IR, we develop digital estimators capable of extracting the resonance frequency and the quality factor. The full electronic architecture is simulated on a mixed-signal environment and implemented on FPGA-based digital hardware. For a specifically emulated sensor, results show that the resonance frequency can be identified and tracked from $3 \mathrm{kHz}$ up to $12 \mathrm{kHz}$ with a Normalized Square Error (NSE) of 1.38E-1. The quality factor is linearized with a correlation coefficient of 0.986 between $Q=1$ to $Q=60$. Those results can be easily scaled to any frequency range by adapting the sampling frequency.
\end{abstract}

Resonant sensor interface, frequency estimation, quality factor estimation, MEMS, LIMBO, resonant sensor.

\section{INTRODUCTION}

Resonant sensors are widely used in many application areas since the mechanical resonant phenomenon can be easily associated with a physical quantity to be measured. The resonance frequency and the quality factor are the common choice to carry sensing information [1]. A shift in these quantities can be used to sense a variety of physical properties such as acceleration, pressure, chemical concentrations, viscosity, angular speed... However, in classical sensing applications, inevitable changes in the operating conditions (temperature, pressure) or in the manufacturing process are detrimental to the nominal sensor operation. They should be electronically detected and compensated for. Most electronic architectures for resonant sensor parameters tracking involve high resolution analog-to-digital converters (ADC) and digitalto-analog converters (DAC) [2,3] requiring long design effort and larger silicon area. The architecture proposed in this article relies on direct digital excitation (instead of the DAC) and binary observation and therefore only requires a comparator instead of a full ADC.

The electronic interface that has been designed includes the least-mean square version of the LIMBO identification method described in [4]. Specific digital estimators have been developed to extract frequency and quality factor from the estimated impulse response. In this article, we present the simulation and FPGA implementation results for an innovative and low cost hardware method for online tracking of the major parameters of a resonant sensor.

\section{ARCHITECTURE DESCRIPTION}

The principle of the proposed architecture is represented on Fig.1. The resonant sensor is directly excited by a digital signal and the sign of its output signal is observed by the mean of a comparator (1-bit ADC). Based on those data the sensor impulse response (IR) can be identified. A convergence estimator monitors that the algorithm has computed the correct estimated IR which can be used for by resonance frequency and quality factor estimators. All the blocks are digital except for the comparator and can be implemented on any digitallogic target such as a Field Programmable Gate Array (FPGA).

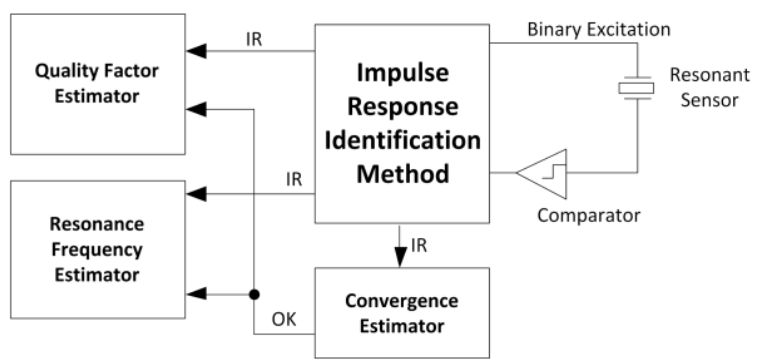

Figure 1. Architecture of the binary-based identification electronic interface for MEMS resonant sensor

\section{Impulse Response Identification Method}

The sensor IR identification method is based on the LIMBO algorithm (Least-mean-square Identification Method based on Binary Observation). The known input signal $u_{n}$ is filtered by a discrete time invariant system $H\left(z^{-1}\right)$ to produce the scalar estimate $\hat{y}_{n}$ of the system output $y_{n}$. $\mathrm{H}$ has a finite impulse response of length $\mathrm{P}$ which can be represented by a column vector $\left.\left(\theta_{k}\right)\right|_{k=1} ^{P}$. At step $n$, this LMS-like algorithm can be written as:

$$
\begin{gathered}
\hat{y}_{n}=\boldsymbol{\theta}_{\boldsymbol{n}}{ }^{\prime} \cdot \boldsymbol{\varphi}_{\boldsymbol{n}-1} \\
\boldsymbol{\theta}_{\boldsymbol{n}}=\boldsymbol{\theta}_{\boldsymbol{n}-\mathbf{1}}-2 \frac{\boldsymbol{\varphi}_{\boldsymbol{n}-1}}{\boldsymbol{\varphi}^{\prime}{ }_{\boldsymbol{n}-1} \boldsymbol{\varphi}_{\boldsymbol{n}-1}}\left|s_{n}-\hat{s}_{n}\right| \hat{y}_{n}
\end{gathered}
$$




$$
\boldsymbol{\varphi}_{\boldsymbol{n}}=\left[\begin{array}{llll}
u_{n} & u_{n-1} & \ldots & u_{n-P}
\end{array}\right]
$$

$\boldsymbol{\varphi}_{\mathbf{n}}$ is the vector of binary stimulus signal, $s_{n}$ is the sign of the output of the sensor and $\hat{s}_{n}$ the sign of its estimation. The only external information required to update the estimated IR $\boldsymbol{\theta}_{\mathbf{n}}$ is the sign of the sensor output signal. For the identification to be accurate, the output signal must contain full information on the sensor's dynamic and therefore the input signal need to be similar as possible to a white noise with a flat power spectral density. As shown in Fig. 2, a 256-bits Linear Feedback Shift Register (LFSR) has been used in this study with a characteristic polynomial given by $x^{256}+x^{241}+$ $x^{178}+x^{121}+1$ [5]. All values are coded on $N$ bits and $\boldsymbol{\theta}(\boldsymbol{n})$ is supported by a $P$ times $N$ bits register based memory.

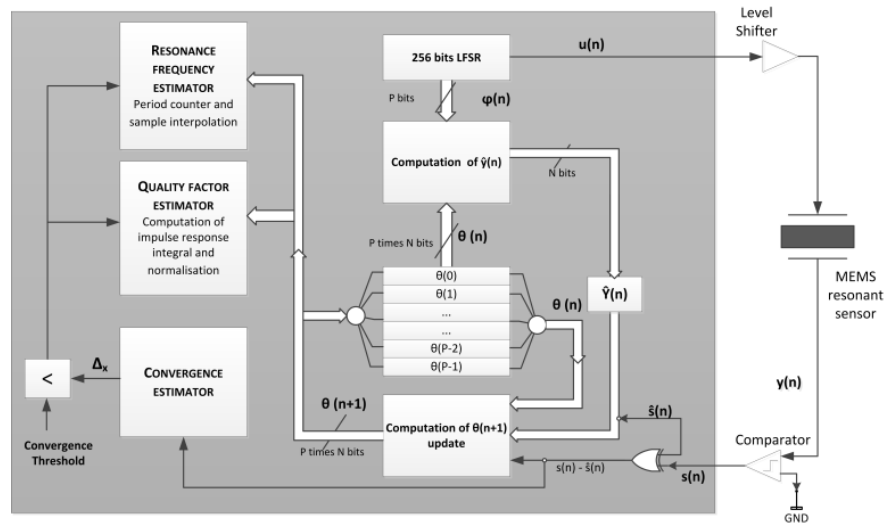

Figure 2. Detailed representation of the LIMBO implementation

\section{Convergence Estimator}

The only error information being the difference of sign between the observed sensor output and the estimated output, a real-time convergence estimator can be defined by:

$$
\Delta_{x}=\sum_{i=k}^{k-P}\left|s_{k}-\hat{s}_{k}\right|
$$

It is the sum of sign differences over the last $\mathrm{P}$ samples processed by the identification algorithm. The Register Transfer Level (RTL) diagram of Fig. 3 details the hardware implementation of $\Delta_{x}$ where a $P$ bits shift register is loaded with the sign difference at each sample and the sum of the sign differences computed by a combinatorial adder tree.

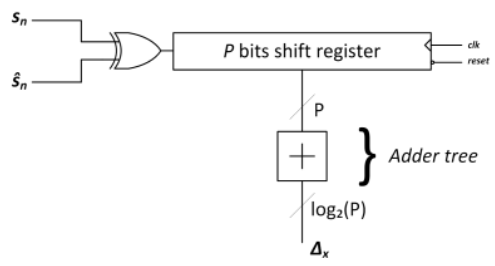

Figure 3. RTL diagram for $\Delta_{\mathrm{x}}$ computation

\section{Resonance Frequency Estimator}

The principle of this estimation is based on counting the number of samples contained in $k$ pseudo-periods of the impulse response. Data interpolation around zero crossing samples of the IR is used to increase the precision of the estimation.

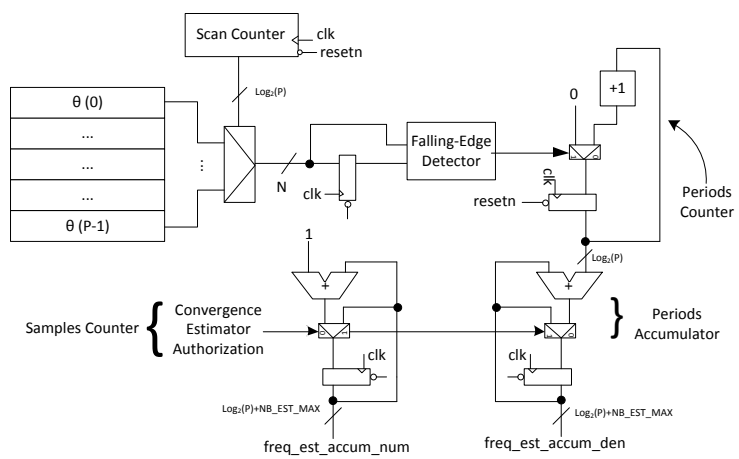

Figure 4. RTL diagram of Resonance Frequency Estimator

On Fig. 4, the RTL diagram of the frequency estimator shows that the IR is stored in a register bank of $\mathrm{P}$ coefficients coded on N-bits. The sign of IR is continuously scanned at sample rate to detect each half period. When authorized by the convergence estimator, the number of sample in a period between two zero-crossing samples is accumulated as well as the number of half-periods.

\section{Quality Factor Estimator}

In order to extract the quality factor (Q) from the estimated impulse response, it is necessary to transform the estimated transfer function in a function of Q. The numerical computation of the area under the IR of a second-order resonator can be written as:

$$
f(Q)=\frac{2 Q w_{0}}{\rho}\left(\sum_{i=1}^{\infty}\left\{\left(\int_{0}^{\pi} \sin (t \rho \gamma) d t\right) e^{-T_{i} \gamma}\right\}\right)
$$

where $w_{0}$ is the resonance frequency, $\rho=\sqrt{\left(4 Q^{2}-1\right)}$, $\gamma=\frac{w_{0}}{2 Q}$ and $T_{i}$ the index of each half-period.

When $Q \gg 10$, the solution of (5) is given by:

$$
f(Q)=\left(4 Q / \sqrt{\left(4 Q^{2}-1\right)}\right) \cdot\left(e^{-3 \pi / 4 Q}-e^{-\pi / 4 Q}\right)^{-1}
$$

Fig. 5 clearly shows that for high values of $Q, f(Q)$ has a linear behavior.

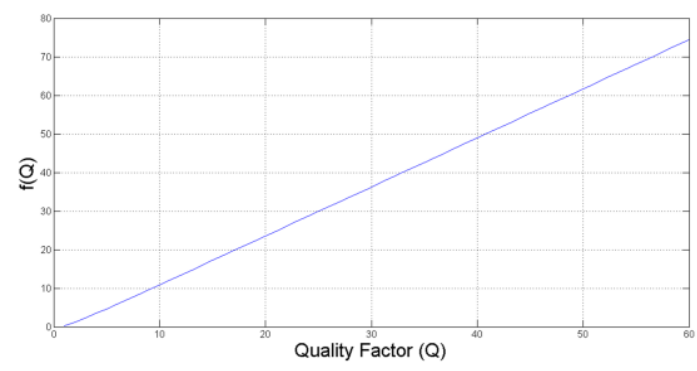

Figure 5. Numerical Estimation of the area of the impulse response as a function of Q

This work was supported by the French National Research Agency (ANR) under contract ANR-08-SEGI-020. 
As shown in Fig.6, the coefficients of the estimated IR are stored in a register-bank which is scanned to compute both the sum of the absolute value of each coefficient and the maximum of the IR. The numerical sum can then be normalized by the gain of the estimated transfer function providing a value proportional to $Q$ at a given resonance frequency.

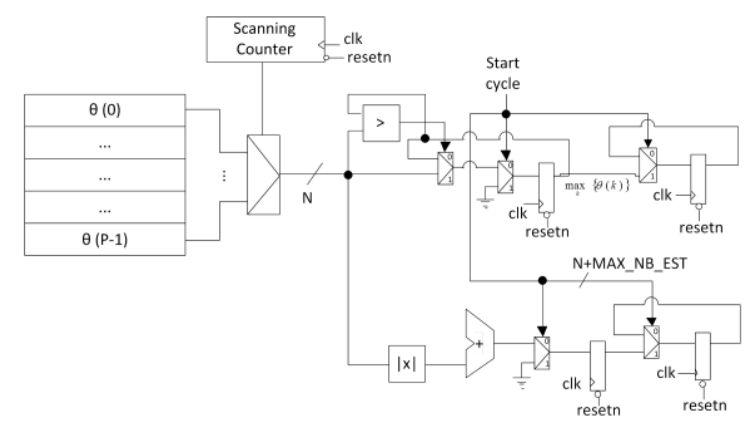

Figure 6. RTL diagram of Quality Factor Estimator

\section{RESULTS}

The architecture was validated and optimized by simulation then implemented on a FPGA based experimental set-up. Results will be detailed in terms of convergence of the algorithm and performance of the estimators.

\section{Simulation Methodology and Experimental Set-Up}

The digital electronics has been described in VHDL and simulated all together with the analog parts of the architecture in CADENCE Virtuoso AMS Designer Environment. The mathematical model of sensor has been translated in VerilogAMS with tunable resonance frequency and quality factor. In respect to the configurability of the LIMBO algorithm, it is possible to modify the length of the estimated impulse response $(\mathrm{P})$, the number of bits used to code each coefficient $(\mathrm{N})$ and the sampling frequency.

The experimental set-up shown in Fig.7 is used to implement our architecture around an Altera Stratix IV FPGA (Terasic DE4 Board). For design flexibility, the MEMS resonant sensor is replaced by a Multiple Feedback (MFB) active filter where the resonance frequency and the quality factor can be adjusted.

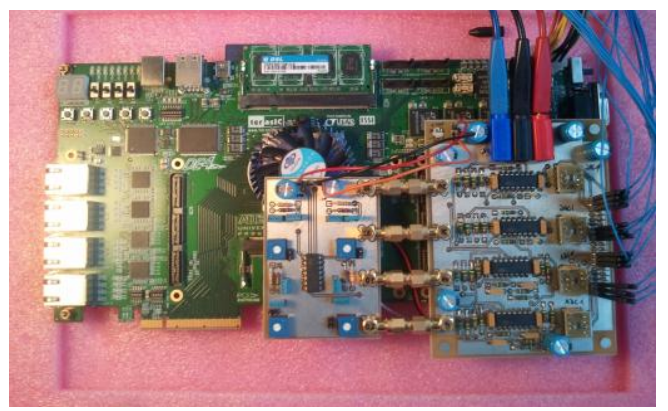

Figure 7. Experimental Set-up showing a digital FPGA development board associated with the analog front end used to emulate a MEMS resonant sensor

\section{LIMBO Identification Algorithm Behavior and Performance}

For a 400ms simulation with $\mathrm{P}=256, \mathrm{~N}=19$ and a sampling frequency of $142 \mathrm{kHz}$, Fig.8 shows the typical evolution of $\Delta_{x}$ over time when identifying a sensor with $\mathrm{Q}=20$ and a $7 \mathrm{kHz}$ resonance frequency. The LIMBO algorithm has converged after only $150 \mathrm{~ms}$ when less than $3 \%$ of the preceding estimation of $y_{n}$ differ in sign with the real output sign.

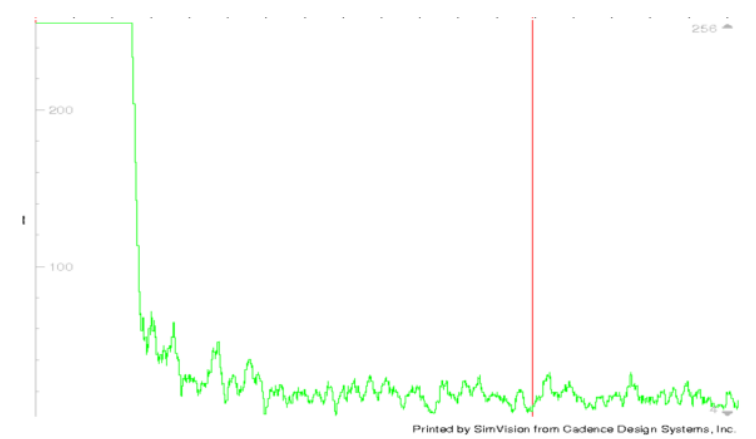

Figure 8. Typical evolution of $\Delta_{\mathrm{x}}$ for $\mathrm{P}=256, \mathrm{~N}=19, \mathrm{~F}_{\text {sampling }}=142 \mathrm{kHz}$.

\section{Resonance Frequency Identification}

After tuning the LIMBO algorithm for an optimal frequency estimation at $7 \mathrm{kHz}$, multiple identification have been performed for resonance frequency ranging from $3 \mathrm{kHz}$ up to $12 \mathrm{kHz}$. The result from the proposed estimator is compared to the Steiglitz-McBride algorithm. This iterative technique for linear systems identification serves as a standard to extract the transfer function from an identified impulse response. As shown in Fig 9, the error of estimation increases as the resonance frequency is far from the optimal $7 \mathrm{kHz}$ value. Nevertheless a maximal normalized square error of 0.138 indicates a correct estimation over the considered frequency range.

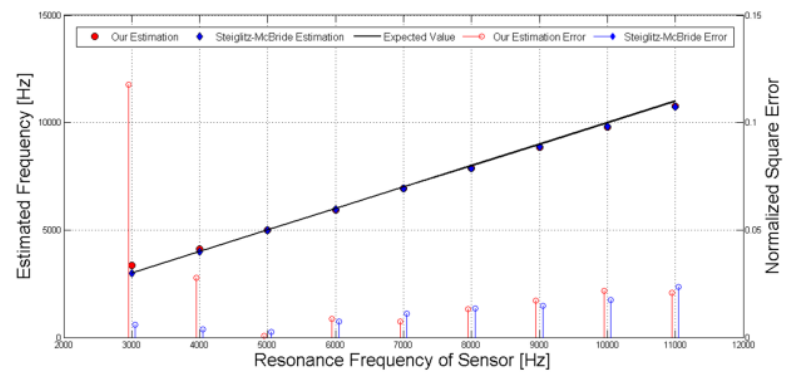

Figure 9. Resonance Frequency Estimation Results $-\mathrm{P}=350, \mathrm{~N}=19$, $\mathrm{F}_{\text {sampling }}=142 \mathrm{kHz}-$ Simulation duration : $400 \mathrm{~ms}$ Sensor Quality Factor : Q=20 


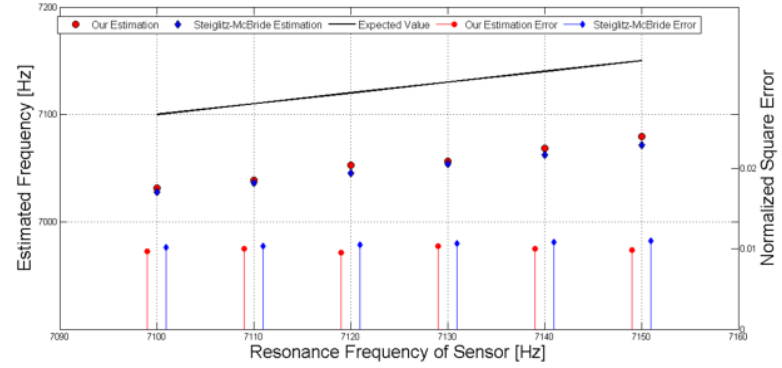

Figure 10. Resonance Frequency Estimation Results $-\mathrm{P}=350, \mathrm{~N}=19$, $\mathrm{F}_{\text {sampling }}=142 \mathrm{kHz}-$ Simulation duration : $400 \mathrm{~ms}-$ Sensor Quality Factor : Q=20

To test the sensitivity of the frequency estimator coupled with the LIMBO identification algorithm, a series of long simulation (1.7 seconds) have been conducted by varying the resonant frequency from $7100 \mathrm{~Hz}$ to $7150 \mathrm{~Hz}$ in steps of $10 \mathrm{~Hz}$. The most interesting result comes from Fig. 10 that shows the ability of the proposed solution to measure a resonance frequency with an NSE of about 0.01. This performance is also compared with the Stieglitz-McBride reference method.

\section{Quality Factor Identification}

Based on the same simulation protocol, the performances of the quality factor estimator have been investigated. For a fixed resonance frequency of $7 \mathrm{kHz}$ and a quality factor ranging from 2 up to 60 , the results of the estimator runs are presented on Fig.10. The quality factor estimation has a higher linearity in the range of 10 to 45 with a correlation coefficient of 0.98602 . In the extended range between 2 and 60, the linear regression presents the Pearson correlation coefficient of 0.87731 . For quality factors lower than 10, the impulse response is short with respect to the sampling frequency and the number of coefficients available to represent the impulse response. For quality factors higher than 50, the impulse response is not entirely observable in the identification windows and the damping behavior is not correctly identified.

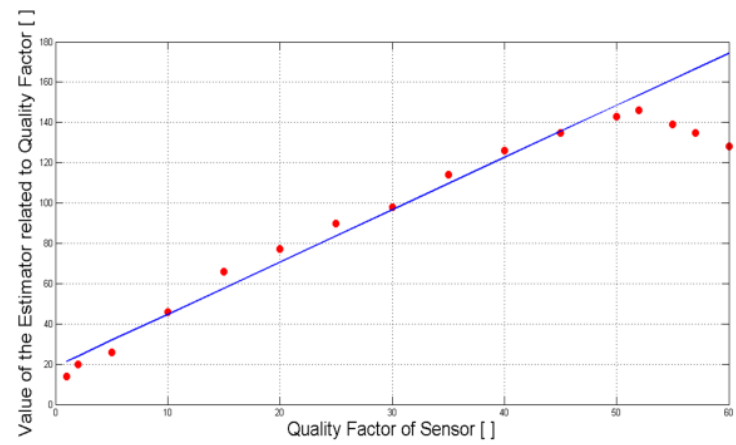

Figure 11. Quality Factor Estimation Results $-\mathrm{P}=350, \mathrm{~N}=19$, Fsampling $=142 \mathrm{kHz}-$ Simulation duration : 400ms - Sensor Resonance Frequency $7 \mathrm{kHz}$

\section{CONCLUSION}

This paper reports the full description of digital architecture that has proven to be able to accurately estimate the resonant frequency and quality factor of a resonant sensor using only binary one-bit input and output. This approach opens the usage of MEMS resonant sensor without the need for complex and costly analog circuit such as ADCs or DACs. The originality of this work rest in the association of a binary least mean square identification algorithm with two specific parametric estimators which have been described in a Hardware Description Language, simulated in a mixed signal environment and finally implemented in a FPGA. A typical MEMS resonant sensor can be identified in $150 \mathrm{~ms}$ when less of $3 \%$ of the estimated output sign differ from the real sensor's output. After successful estimation of the impulse response the resonance frequency has been identified from $3 \mathrm{kHz}$ up to $12 \mathrm{kHz}(\mathrm{NSE}=0.0138)$ as well as the quality factor from $\mathrm{Q}=1$ up to $\mathrm{Q}=60$. Further work will concentrate on the identification and the design of new estimators for other type of MEMS sensors such as nonresonant micro-wire temperature and pressure transducers.

\section{REFERENCES}

[1] M. Hoummady and D. Hauden. Acoustic wave thermal sensitivity: Temperature sensors and temperature compensation in microsensors. Sensors and Actuators A, 44(3):177-182, September 1994

[2] Wei Dong and al., "Research on MEMS acceleration Sensor Dectection Circuit based on Adaptive Filter", Proceedings of the 2009 IEEE International Conference on Mechatronics and Automation, p. 14331437, 2009.

[3] J. K. Sella, A. O. Niedermayera, B. Jakobya, "Digital phase-locked loop circuit for driving resonant sensors", Procedia Engineering 5, p. 204 207, 2012.

[4] Jérôme Juillard, Eric Colinet, "Initialization of the BIMBO self-test method using binary inputs and outputs", Proceedings of the 46th IEEE Conference on Decision and Control, p. 578-583, 2007.

[5] Miodrag Zivkovic, "Generation of primitive binary polynomials", International Conference on Algebra, Logic and Discrete Mathematics, April 14-16, 1995. 